\title{
L-FABP and I-FABP expression in newborn rats changes inversely in the model of necrotizing enterocolitis ${ }^{1}$
}

\author{
Luís Felipe Tsuyoshi Mitidiero', Ana Leda Bertoncini Simões ${ }^{\text {II }}$, Frances Lilian Lanhellas Gonçalves ${ }^{\text {III }}$, Rebeca Rodrigues Lopes \\ Roslindo Figueira $^{\text {IV }}$, Orlando Castro e Silvav ${ }^{\text {, Lourenço Sbragia }}{ }^{\text {II }}$
}

DOI: http://dx.doi.org/10.1590/S0102-8650201400140009

${ }^{I}$ Medical Student, Ribeirão Preto Medical School, University of Sao Paulo - USP, Ribeirão Preto - SP, Brazil. Acquisition of data, data analysis and manuscript writing.

"RN, Master's degree, Doctoral degree Fellow, Division of Pediatric Surgery, Department of Surgery and Anatomy, Ribeirão Preto Medical School, University of Sao Paulo - USP, Ribeirão Preto - SP, Brazil. Acquisition of data, data analysis and manuscript writing;

IIIPhD, Post-Doctoral degree Fellow, Division of Pediatric Surgery, Department of Surgery and Anatomy, Ribeirão Preto Medical School, University of Sao Paulo - USP, Ribeirão Preto - SP, Brazil. Acquisition of data, data analysis and manuscript writing.

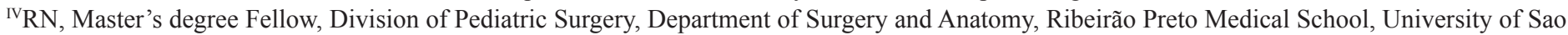
Paulo - USP, Ribeirão Preto - SP, Brazil. Acquisition of data and data analysis.

${ }^{v} \mathrm{MD}, \mathrm{PhD}$, Full Professor, Chairman and Head of the Department of Surgery and Anatomy, Ribeirão Preto Medical School, Sao Paulo University (USP), Ribeirão Preto-SP, Brazil. Data analysis.

${ }^{\mathrm{VI}} \mathrm{MD}, \mathrm{PhD}$, Associate Professor, Division of Pediatric Surgery, Department of Surgery and Anatomy, Ribeirão Preto Medical School, University of Sao Paulo - USP, Ribeirão Preto - SP, Brazil. Mentor, conception and design of the study, manuscript writing and critical revision.

\section{ABSTRACT}

PURPOSE: To determine the expression of hepatic L-FABP and intestinal I-FABP in an experimental model of necrotizing enterocolitis (NEC) in neonatal rats.

METHODS: Newborn Sprague-Dawley rats were divided into four groups: Control (C1) - exclusive breastfeeding at the first and sixth procedures (C6), NEC1 - fed formula milk and submitted to hypoxia and hypothermia at the first and sixth procedures (NEC6). The newborn pups were fed twice a day for three days, for a total of six procedures. Samples were collected for morphometric evaluation (body weight, liver weight, liver weight/body weight ratio, intestinal weight and intestinal/body weight ratio) and for immunohistochemical and Western blotting analysis. The values obtained were analyzed statistically, with the level of significance set at $p<0.05$.

RESULTS: Morphometric measurements showed reduction of body and liver weights in the NEC group $(p<0.05)$. Both immunohistochemistry and western blotting revealed that L-FABP expression in the liver was decreased and I-FABP expression in the ileum was increased in the NEC group $(p<0.05)$.

CONCLUSION: L-FABP and I-FABP expression changed inversely in the rat NEC model. These findings can contribute to a better diagnosis of NEC in human newborns.

Key words: Enterocolitis, Necrotizing, L-FABP, I-FABP, liver, intestine, rats. 


\section{Introduction}

Necrotizing enterocolitis (NEC) is a severe intestinal inflammatory disease affecting neonates, with a higher incidence among premature babies. The disease is characterized by intestinal necrosis and leads to multiple organ failure ${ }^{1}$. The mortality rate associated with NEC ranges from 20 to $30 \%$, with gastrointestinal sequelae such as stenosis and short bowel syndrome possibly being present in cases in which there is resolution or the need for surgical intervention ${ }^{2}$. The physiopathological events of the disease are still unknown, but their origin appears to be multifactorial. Prematurity, bacterial infection, intestinal hypoxia-ischemia and enteral feeding are the major risk factors of the disease ${ }^{3}$.

In severe cases of NEC, in addition to intestinal involvement there is a frequent occurrence of injury to one or more organs resulting in pulmonary, renal and/or hepatic failure 4 . Studies have reported significant pathological changes in hepatic morphology and in hepatobiliary function in patients with NEC, especially those submitted to parenteral nutrition ${ }^{5}$.

In the experimental NEC model created by Barlow and Santulli ${ }^{6}$ and later modified by Caplan et $a .^{7}$, the disease is induced by using enteral formula feeding associated with intermittent episodes of hypoxia and hypothermia.

Molecular markers are being used for the detection of injuries mainly in the intestine and liver, such as intestinal or liver fatty acid binding protein (I-FABP or L-FABP). I-FABP is found in the cytoplasm of small bowel enterocytes and L-FABP is found in the cytoplasm of hepatic, intestinal, renal and gastric cells. Both proteins act on the uptake and intracellular transport of fatty acids and belong to a family of nine other FABPs. They are characterized by being water soluble, of low molecular weight (14-15 kDa), stable, easily released into the circulation, filtered through the glomeruli, and being promptly detected in urine. Some studies have investigated the relation of serum L-FABP levels with NEC, but no studies are available about their tissue expression in this disease ${ }^{8}$.

The elucidation of physiopathological mechanisms and the discovery of a treatment or method for an early diagnosis of NEC have been a challenge in the hope to reduce the high morbidity and mortality rates of the disease. In this respect, finding markers of tissue injury that may correlate with the disease could be of benefit for these patients. Thus, the objective of the present study was to determine the expression of L-FABP in the liver and compare it to the expression of I-FABP in the intestine of newborn rats submitted to the experimental NEC model.

\section{Methods}

The project was approved by the Ethics Committee for Animal Experimentation of the Ribeirão Preto Medical School, University of São Paulo (protocol \# 040/2011). Male and female adult Sprague-Dawley rats were maintained under controlled lighting conditions (12 hours of light/12 hours of dark), at a mean temperature of $23^{\circ} \mathrm{C}$, relative humidity close to $55 \%$, with free access to water and rat chow. The animals were mated during the dark period of the cycle, with two females being placed in the cage of a male, and day zero of pregnancy was determined by the presence of sperm in vaginal smears from females in estrus (pregnancy of 22 days).

\section{Experimental groups}

All pups were weighed at birth and allotted at random to two experimental groups: Control (C), in which the animals were not manipulated and were suckled by their respective dams, and Necrotizing Enterocolitis (NEC), in which the pups were removed from their dams immediately after birth, weighed daily, fed artificial Esbilac ${ }^{\circledR}$ milk (PetAg, Hampshire, IL, USA) ( 200 $\mathrm{kcal} / \mathrm{kg} /$ day) and subjected to an ischemia/hypothermia protocol twice a day for a total of six days until sacrifice.

These groups were divided into two subgroups $(n=4)$ : collection 1 (animals sacrificed after the first ischemia/ hypothermia process) - groups $\mathrm{C} 1$ and NEC1, and collection 6 (animals sacrificed after 6 ischemia/hypothermia processes) groups C6 and NEC6. Control groups C1 and C6 were sacrificed at the same times as the experimental groups but were not submitted to any procedure.

\section{Surgical procedure}

The pups were weighed and sacrificed by decapitation. After laparotomy, the liver and ileum were collected, weighed and fixed in $10 \%$ buffered formol.

\section{Morphometric evaluation}

Body weight (BW), liver weight (LW), intestinal weight (IW) and the LW/BW and IW/BW ratios were used to analyze morphological differences between groups. 


\section{Processing for L-FABP and I-FABP immunohistochemistry}

After fixation, the samples were dehydrated in a growing ethanol series from 70 to $100 \%$, cleared in xylene and embedded in histological paraffin. Crosswise $5-\mu \mathrm{m}$ histological sections of the liver and intestine segments were obtained with a Leica microtome model RM 2145 and later collected on histological slides presilanized for better adherence.

\section{Immunohistochemical analysis of L-FABP and I-FABP}

Immunohistochemistry was used to locate tissue proteins. Sections of $3 \mu \mathrm{m}$ were mounted on slides which were then deparaffinized with xylene and hydrated in a growing ethanol series. The sections were treated with $\mathrm{H}_{2} \mathrm{O}_{2}(30 \%) 3 \%$ in methanol for 10 minutes at room temperature and then in $50 \mathrm{mM}$ Tris- $\mathrm{HCl}$, $\mathrm{pH} 9.5$, containing 5\% urea for antigen retrieval under steam, and then washed with phosphate buffer saline (PBS - 20 $\left.\mathrm{mM} \mathrm{Na} \mathrm{HPO}_{4}+0.45 \mathrm{M} \mathrm{NaCl}, \mathrm{pH} 7.4\right)$. The slides were incubated in $10 \%$ rabbit block solution diluted with PBS for 30 minutes in order to block nonspecific binding sites. Intestinal and hepatic sections were incubated with goat anti-I-FABP, sc-16063 and rabbit anti-FABP-L, sc-50380, respectively (Santa Cruz Biotechnology, Santa Cruz, CA, USA) diluted 1:200 with bovine serum albumin (BSA) at $4{ }^{\circ} \mathrm{C}$ overnight. After washing and removal of the primary antibody, the appropriate secondary antibodies were added to the hepatic and intestinal sections (sc-2004 and sc-2768, respectively, Santa Cruz Biotechnology) diluted 1:200 in BSA for two hours. For the negative control, the primary antibody was omitted. The histochemical reaction was visualized using the Vectastain $\mathrm{ABC}$ kit (Vector Labs, Burlingame, CA, USA) and 3,3'-diaminobenzidinetetra-hydrochloride (Sigma, St Louis, MO, USA). Finally, the slides were counterstained with Harris hematoxylin, dehydrated in a growing alcohol series and xylene, and covered with coverslips mounted with Permount ${ }^{\circledR}$ (Fisher Scientific, Pittsburgh, PA, USA).

\section{Western blotting analysis of L-FABP and I-FABP}

Prepared samples of intestine and liver homogenates from each group containing $20 \mu \mathrm{g}$ protein were applied to $1 \%$ polyacrylamide gel containing $0.1 \%$ sodium lauryl sulfate (SDS-PAGE) and running buffer. Electrophoresis was carried out at a constant current intensity of $100 \mathrm{~V}$ for approximately 2 hours and 30 minutes. The protein bands were then transferred electrophoretically to a nitrocellulose membrane in transfer buffer at $120 \mathrm{~V}$ and $4^{\circ} \mathrm{C}$ for 90 minutes. Nonspecific sites for primary antibody binding to the membrane were blocked by membrane incubation with a $5 \%$ solution of Molico skim milk in 0.01 M PBS buffer, $\mathrm{pH} 7.4$, under constant shaking for one hour. The membranes were then incubated for 12 hours at $4{ }^{\circ} \mathrm{C}$ with the same primary antibodies used for immunohistochemistry (I-FABP (sc-16063) and L-FABP (sc-50380)) diluted 1:100 in $3 \% \mathrm{PBS} / \mathrm{BSA}$

After incubation, the membranes were washed with $0.01 \mathrm{M}$ PBS buffer, $\mathrm{pH} 7.4$, and incubated with the same secondary antibodies used for immunohistochemistry (I-FABP (sc-2004) and L-FABP (sc-2768)) diluted 1:2000 in 3\% PBS/ BSA for two hours. The membranes were then visualized by chemiluminescence (Pierce, USA) for 5 minutes and developed with the photodocumentation system ChemiDoc ${ }^{\mathrm{TM}} \mathrm{XRS}+$ System (Bio-Rad, USA), and the bands were analyzed and measured using the Image $\mathrm{Lab}^{\mathrm{TM}}$ software.

\section{Statistical analysis}

Morphology and molecular biology data were analyzed by one-way ANOVA followed by the Tukey-Kramer post-test and immunohistochemical data were analyzed by the Kruskal-Wallis test followed by the Dunn post-test, with the level of significance set at $p<0.05$. Data are reported as mean \pm standard deviation. All analyses were carried out using the GraphPad Prism 5.0 software (GraphPad Software Inc., La Jolla, CA, USA).

\section{Results}

\section{Morphometric analysis}

Evaluation of BW revealed weight gain in the pups of the Control group (C), with a significant difference between $\mathrm{C} 1$ and C6 $(p<0.001)$. BW was significantly reduced in the NEC6 group compared to $\mathrm{C} 1(p<0.001)$, to C6 $(p<0.001)$ and to NEC1 $(p<0.001)$. LW was reduced in group NEC6 compared to $\mathrm{C} 1, \mathrm{C} 6$ and NEC1 $(p<0.001)$. IW was increased in group C6 compared to groups $\mathrm{C} 1$, NEC1 and NEC6 $(p<0.001)$. The LW/BW ratio was reduced in group C6 compared to groups C1 and NEC1 $(p<0.001)$. There was also a reduction in group NEC1 compared to $\mathrm{C} 1(p<0.05)$ and in group NEC6 compared to C1 and NEC1 $(p<0.001)$. The IW/BW ratio was increased in group C6 compared to groups $\mathrm{C} 1$ and $\mathrm{NEC} 1$ $(p<0.001)$ and also in group NEC6 compared to groups C1, NEC1 and C6 ( $p<0.001, p<0.001$ and $p<0.05$, respectively) (Table 1). 
TABLE 1 - Morphometric analysis of body weight (BW), liver weight (HW), intestinal weight (IW), LW/BW ratio and IW/BW ratio. Data are reported as mean \pm standard deviation.

\begin{tabular}{lccccc}
\hline Groups & BW & IW & LW & IW/BW & LW/BW \\
\hline C1 & $5678( \pm 684)$ & $201( \pm 27)$ & $294( \pm 47)$ & $0.0371( \pm 0.0014)$ & $0.0517( \pm 0.0040$ \\
NEC1 & $6519( \pm 164)^{*_{a}}$ & $242( \pm 30)$ & $303( \pm 17)$ & $0.0368 \pm 0.0041)$ & $0.0465)( \pm 0.0021)^{*_{a}}$ \\
C6 & $8809( \pm 528)^{* \text { ab }}$ & $449( \pm 74)^{* *_{\text {ab }}}$ & $322( \pm 30)$ & $0.0486( \pm 0.0044)^{* *_{\text {ab }}}$ & $0.0366( \pm 0.0018)^{* \text { ab }}$ \\
NEC6 & $4521( \pm 272)^{* *_{\text {abc }}}$ & $238( \pm 16)^{* *_{\mathrm{c}}}$ & $177( \pm 19)^{* *_{\text {abc }}}$ & $0.0534( \pm 0.0034)^{* *_{\text {ab }} *_{\mathrm{c}}}$ & $0.0393( \pm 0.0042)^{* *_{\mathrm{ab}}}$ \\
\hline
\end{tabular}

a: compared to $\mathrm{C} 1$; b: compared to NEC1; c: compared to C6; d: compared to NEC6 *p<0.05**p<0.001

\section{Immunohistochemical analysis}

Immunohistochemical study of L-FABP expression revealed increased protein labeling in group C6 compared to group NEC1 $(p<0.001)$. On the other hand, there was a significant reduction $(p<0.001)$ of expression in group NEC6 compared to groups C1, C6 and NEC1 $(p<0.001)$ (Figure 1). Intestinal I-FABP labeling was increased in group NEC6 compared to the other three groups $(p<0.001)$ (Figure 2).
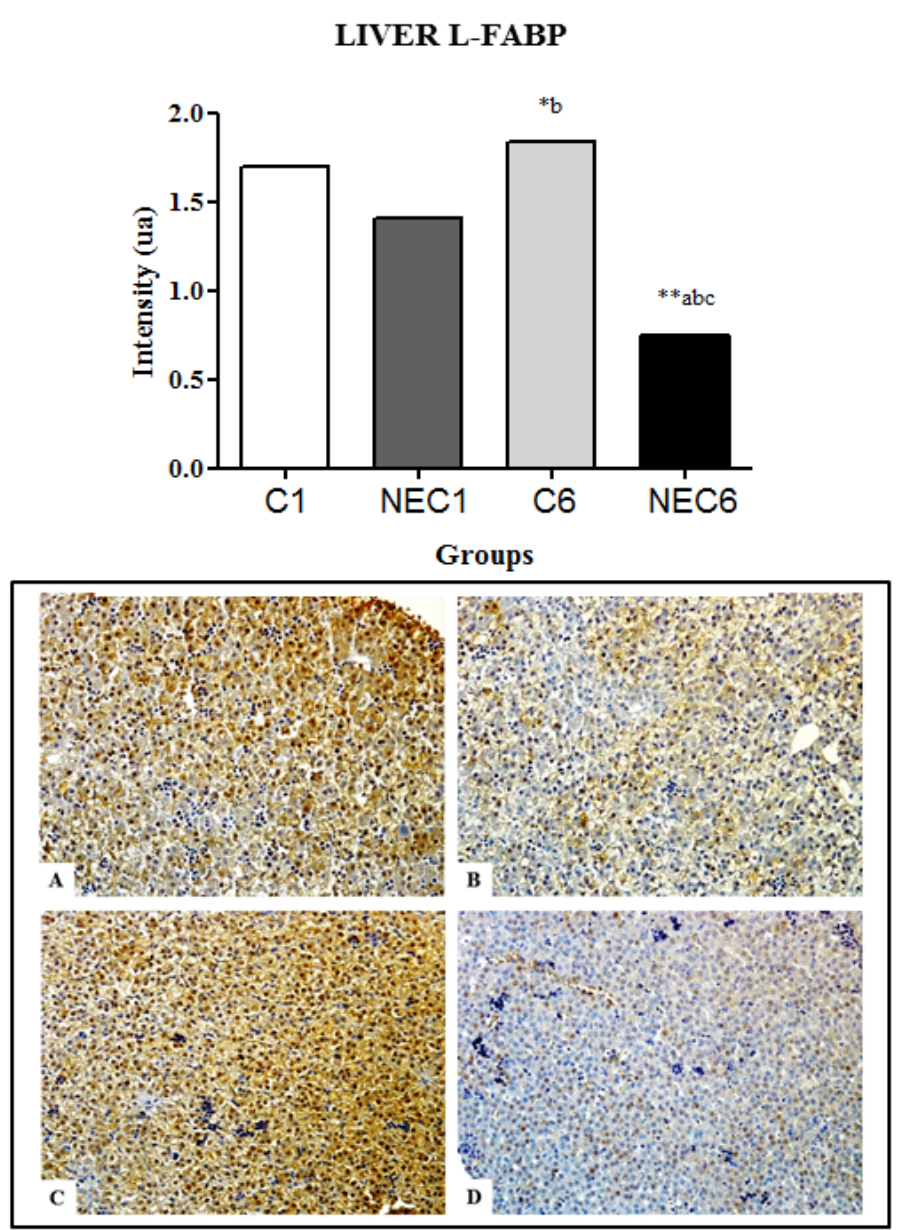

FIGURE 1 - L-FABP expression in the liver after immunohistochemistry. A) Group C1; B) Group NEC1; C) Group C6; D) Group NEC6. Strong labeling is observed in the control groups and low expression in the organ of a newborn pup with NEC. a: compared to $\mathrm{C} 1$; $\mathbf{b}$ : compared to NEC1; $\mathbf{c}$ : compared to C6; d: compared to NEC6, ${ }^{*} p<0.05 ; * * p<0.001$.
ILEUM I-FABP
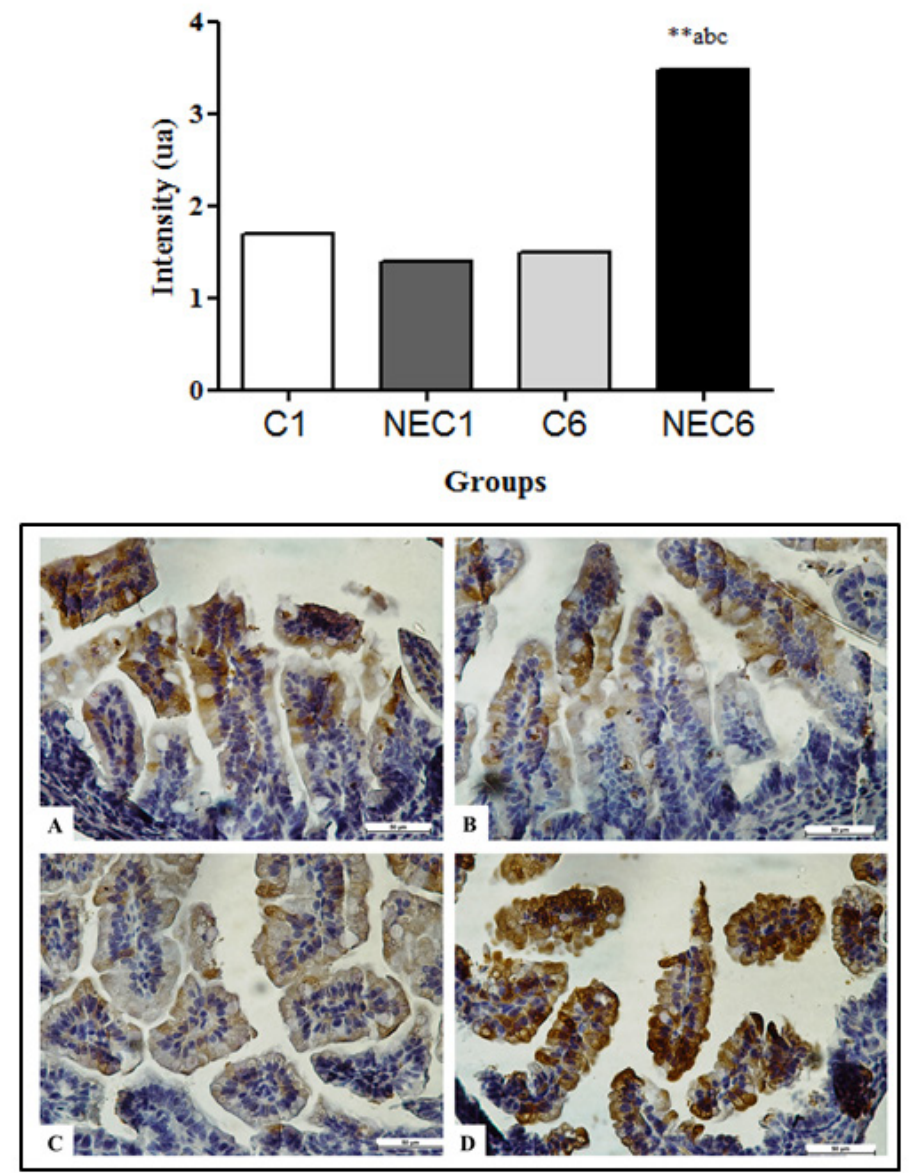

FIGURE 2 - I-FABbexpression in the intestine after immunohistochemistry. A) Group C1; B) Group NEC1; C) Group C6; D) Group NEC6. Weak labeling is observed in the control groups and high expression in the organ of a newborn pup with NEC. a: compared to $\mathrm{C} 1$; $\mathbf{b}$ : compared to NEC1; $\mathbf{c}$ : compared to $\mathrm{C} 6, * * p<0.001$.

\section{Western blotting analysis}

The evaluation of L-FABP expression by Western blotting revealed an increase in group C6 compared to groups C1 and NEC1 $(p<0.001)$. Conversely, there was a reduction of density in group NEC6 compared to groups C1, C6 and NEC1 $(p<0.05, p<0.001$ and $p<0.001$, respectively). In the ileum there was an increase in I-FABP expression in group NEC1 compared to group C1 $(p<0.001)$, as well as in group C6 compared to groups $\mathrm{C} 1(p<0.001)$ and NEC1 $(p<0.001)$. In group NEC6 there was an increase in I-FABP expression compared to the other three groups $(p<0.001)$ (Figure 3$)$. 
A

LIVER L-FABP

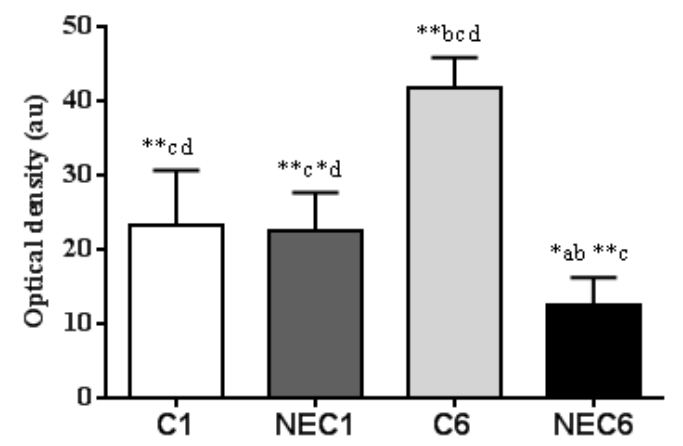

Groups

$14 \mathrm{KDa}$
B

ILEUM I-FABP

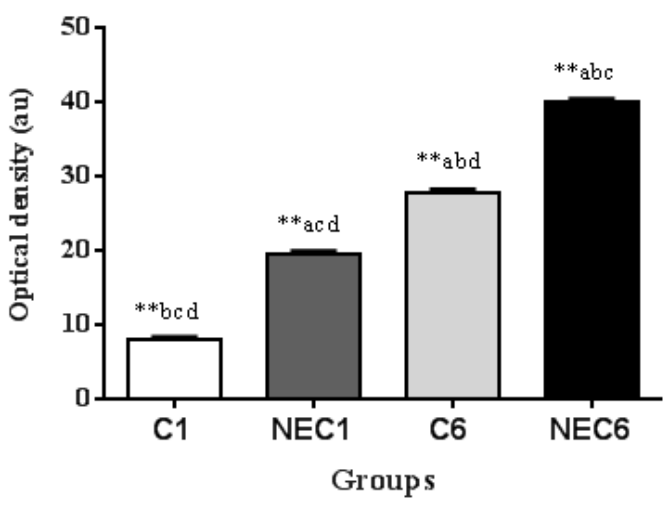

$15 \mathrm{KDa}$

FIGURE 3 - L-FABP expression in the liver and I-FABP expression in the ileum after western blotting. A) Group C1; B) Group NEC1; C) Group C6; D) Group NEC6. Weak labeling is observed in the control groups and high expression in the organ of a newborn pup with NEC. a: compared to C1; b: compared to NEC1; c: compared to C6; d: compared to NEC6. ${ }^{*} p<0.05 ;{ }^{* *} p<0.001$

\section{Discussion}

This was the first study investigating L-FABP expression in the liver in an experimental model of NEC. Several studies on the FABP family and its correlation with intestinal lesions have been conducted, but studies on the association of the expression of these proteins with the liver are still scarce. NEC mainly affects regions of the distal ileum and colon and is characterized by intense inflammatory hemorrhagic necrosis. Changes in hepatic morphology and hepatobiliary physiology also occur in addition to the intestinal involvement ${ }^{4,5}$. Its physiopathology has not been fully clarified, but prematurity, intestinal ischemia, bacterial colonization and enteral feeding are factors hat favor the development of the disease ${ }^{3}$. In the present study, we used as an experimental model the protocol of ischemia and hypothermia associated with enteral formula feeding.

Morphometric analysis revealed a considerable impact of artificial milk feeding on the evolution of BW, with a significant reduction in the groups fed Esbilac $\AA$ artificial milk for a longer period of time. Several other studies using similar milk formulas also reported a weight loss ${ }^{9,10}$.

There was a reduction of LW in the group receiving formula, although the NEC6 group showed significant liver involvement compared to groups $\mathrm{C} 1, \mathrm{C} 6$ and NEC1. The pups submitted to the protocol showed varying degrees of inflammation in the intestinal loops, with NEC6 also showing IW loss. Since the portal vein drains the blood circulation from the intestine directly into the liver, the proinflammatory cytokines originating from the intestinal loops are believed to also cause an inflammatory reaction in the liver, with a deleterious effect on $\mathrm{LW}^{11}$. The $\mathrm{LW} / \mathrm{BW}$ ratio was lower in the NEC6 group than in the C1, C6 and NEC1 groups. Although we did not determine tissue or serum cytokines, we may infer that there was liver damage as a consequence of the intestinal inflammatory process, which also explained the reduction of the IW/BW ratio mainly in the NEC6 group ${ }^{11}$.

Several studies have described the applicability of the L-FABP protein to the detection of hepatic injury in diseases such as hepatocellular adenoma ${ }^{12}$ and chronic HCV infection ${ }^{13}$. In addition, I-FABP has already been studied in experimental and clinical NEC models ${ }^{14}$. In the present study we observed reduced L-FABP labeling in the immunohistochemistry of liver tissue and in Western blotting in pups submitted to the NEC protocol. Memon et al. ${ }^{15}$ demonstrated that bacterial lipopolysaccharide (LPS) and proinflammatory cytokines such as TNF- $\alpha$ and IL$1 \beta$ reduce the content of L-FABP and its mRNA in the liver. In NEC, inflammatory factors released in situ in the liver by Kupffer cells, as well as intestinal cytokines and bacterial factors arriving through the hepatic portal vein must cause inflammation, with a consequent reduction of L-FABP expression ${ }^{11}$.

During infectious and inflammatory processes, the host response is characterized by various infectious changes in lipid metabolism such as increased lipogenesis and fatty acid re-esterification in the liver and inhibition of hepatic fatty acid oxidation $^{16,17}$. Since L-FABP acts on fatty acid metabolism, it may 
be hypothesized that, in an inflammatory disease such as NEC, its expression is reduced in order to contribute to lower fatty acid oxidation. However, it is important to point out that it is not possible to establish a causal relationship.

Studies have demonstrated that L-FABP is present at high serum concentrations in the early stage of NEC, demonstrating that this protein could be a parameter for the early detection of the disease ${ }^{18}$. $\mathrm{Ng}$ et al. ${ }^{19}$ observed that plasma L-FABP levels were higher in premature newborns submitted to surgical intervention for NEC and in those who died due to hepatic portal vein disease. Considering that L-FABP concentration is about six-fold higher in the liver than in the intestine, we may assume that this protein released through a hepatic pathway plays an important role in the detection of NEC. In the present study we observed that the animals submitted to the experimental model of the disease also manifested changes in L-FABP production. The low expression of L-FABP in immunolabeling and in the band density detected by Western blotting may possibly be characterized by the rapid release of the protein into the bloodstream since, by being a low molecular weight protein, it is rapidly filtered through the kidneys, being maintained in relatively low concentrations in blood in physiological situations ${ }^{20}$.

In addition, this hypothesis is supported by studies that have correlated L-FABP with other inflammatory liver diseases. High serum concentrations of this protein have been detected in patients with chronic hepatitis $\mathrm{C}$ and with non-alcoholic fatty liver disease, with the quantity of the molecule showing a good correlation with the degree of hepatic inflammation in both $\operatorname{cases}^{13}$.

In the present study we compared these results with I-FABP expression in the intestine. The expression of this protein was inverse to that of L-FABP, i.e., it was increased mainly in NEC6 and, for the same reason, we believe that this was due to the inflammatory process. After enterocyte rupture, I-FAPB is released into the bloodstream and, due to this high expression, its serum level is increased. All analyses were carried out on intact bowels.

\section{Conclusion}

The hepatic expression of L-FABP is altered in NEC and that this result is inverse to the expression of I-FABP detected in the intestine, data that could contribute to the use of FABPs for a better diagnosis and an improvement of the severity of NEC in affected neonates.

\section{References}

1. Lin PW, Stoll BJ. Necrotising enterocolitis. Lancet. 2006 Oct 7;368(9543):1271-83.

2. Fitzgibbons SC, Ching Y, Yu D, Carpenter J, Kenny M, Weldon C, Lillehei C,Valim C, Horbar JD, Jaksic T. Mortality of necrotizing enterocolitis expressed by birth weight categories. J Pediatr Surg. 2009 Jun;44(6):1072-5; discussion 1075-6.

3. Caplan MS, Jilling T. New concepts in necrotizing enterocolitis. Curr Opin Pediatr. 2001 Apr;13(2):111-5.

4. Morecroft JA, Spitz L, Hamilton PA, Holmes SJ. Necrotizing enterocolitis--multisystem organ failure of the newborn? Acta Paediatr Suppl. 1994;396:21-3.

5. Moss RL, Das JB, Raffensperger JG. Necrotizing enterocolitis and total parenteral nutrition-associated cholestasis. Nutrition. 1996 May;12(5):340-3.

6. Barlow B, Santulli TV. Importance of multiple episodes of hypoxia or cold stress on the development of enterocolitis in an animal model. Surgery. 1975 May;77(5):687-90.

7. Caplan MS, Hedlund E, Adler L, Hsueh W. Role of asphyxia and feeding in a neonatal rat model of necrotizing enterocolitis. Pediatr Pathol. 1994 Nov-Dec;14(6):1017-28.

8. Zimmerman AW, Veerkamp JH. New insights into the structure and function of fatty acid-binding proteins. Cell Mol Life Sci. 2002 Jul;59(7):1096-116.

9. Guven A, Gundogdu G, Vurucu S, Uysal B, Oztas E, Ozturk H, Korkmaz A. Medical ozone therapy reduces oxidative stress and intestinal damage in an experimental model of necrotizing enterocolitis in neonatal rats. J Pediatr Surg. 2009 Sep;44(9):1730-5.

10. Zani A, Eaton S, Leon FF, Malerba A, Hall NJ, De Coppi P, Smith VV, Pierro A. Captopril reduces the severity of bowel damage in a neonatal rat model of necrotizing enterocolitis. J Pediatr Surg. 2008 Feb;43(2):308-14.

11. Halpern MD, Holubec H, Dominguez JA, Meza YG, Williams CS, Ruth MC, McCuskey RS, Dvorak B. Hepatic inflammatory mediators contribute to intestinal damage in necrotizing enterocolitis. Am J Physiol Gastrointest Liver Physiol. 2003 Apr;284(4):G695-702.

12. Fonseca S, Hoton D, Dardenne S, Annet L, Hubert C, Godecharles S, Jouret-Mourin A, Reding R, Otte JB, Rahier J, Gigot JF, Sempoux C. Histological and immunohistochemical revision of hepatocellular adenomas: a learning experience. Int J Hepatol. 2013;2013:398308.

13. Akbal E, Köklü S, Koçak E, Cakal B, Güneș F, Başar O, Tuna Y, Senes M. Liver fatty acid-binding protein is a diagnostic marker to detect liver injury due to chronic hepatitis $\mathrm{C}$ infection. Arch Med Res. 2013 Jan;44(1):34-8.

14. Caplan MS, Miller-Catchpole R, Kaup S, Russell T, Lickerman M, Amer M, Xiao Y, Thomson R Jr. Bifidobacterial supplementation reduces the incidence of necrotizing enterocolitis in a neonatal rat model. Gastroenterology. 1999 Sep;117(3):577-83.

15. Memon RA, Bass NM, Moser AH, Fuller J, Appel R, Grunfeld C, Feingold KR. Down-regulation of liver and heart specific fatty acid binding proteins by endotoxin and cytokines in vivo. Biochim Biophys Acta. 1999 Aug 25;1440(1):118-26.

16. Bagby GJ, Corll CB, Martinez RR. Triacylglycerol kinetics in endotoxic rats with suppressed lipoprotein lipase activity. Am J Physiol. 1987 Jul;253(1 Pt 1):E59-64.

17. Spitzer JJ, Bagby GJ, Mészáros K, Lang CH. Alterations in lipid and carbohydrate metabolism in sepsis. JPEN J Parenter Enteral Nutr. 1988 Nov-Dec;12(6 Suppl):53S-8S.

18. Guthmann F, Börchers T, Wolfrum C, Wustrack T, Bartholomäus $\mathrm{S}$, Spener F. Plasma concentration of intestinal- and liver-FABP in neonates suffering from necrotizing enterocolitis and in healthy preterm neonates. Mol Cell Biochem. 2002 Oct;239(1-2):227-34. 
19. Ng EW, Poon TC, Lam HS, Cheung HM, Ma TP, Chan KY, Wong RP, Leung KT, Lam MM, Li K, Ng PC. Gut-associated biomarkers L-FABP, I-FABP, and TFF3 and LIT score for diagnosis of surgical necrotizing enterocolitis in preterm infants. Ann Surg. 2013 Dec;258(6):1111-8.

20. Pelsers MM, Hermens WT, Glatz JF. Fatty acid-binding proteins as plasma markers of tissue injury. Clin Chim Acta. 2005 Feb;352(12):15-35.

\section{Correspondence:}

Prof. Dr. Lourenço Sbragia

Disciplina de Cirurgia Pediátrica

Departamento de Cirurgia e Anatomia-USP

Avenida Bandeirantes, 3900

14049-900 Ribeirão Preto - SP Brasil

Tel.: (55 16)3602-2593 / 3602-2501

Fax: (55 16)3633-0836

orlandojr@usp.br

${ }^{1}$ Research performed at Laboratory of Experimental Fetal Surgery, Ribeirao Preto Medical School, University of Sao Paulo (USP), Brazil. Mentor: Lourenço Sbragia. 